\title{
Combination of Attributes in Stereovision Matching for Fish-Eye Lenses in Forest Analysis
}

\author{
P. Javier Herrera ${ }^{1}$, Gonzalo Pajares ${ }^{1}$, María Guijarro ${ }^{2}$, J. Jaime Ruz ${ }^{3}$, \\ and Jesús M. De la Cruz ${ }^{3}$ \\ ${ }^{1}$ Dpto. Ingeniería del Software e Inteligencia Artificial, Facultad de Informática, \\ Universidad Complutense, 28040 Madrid, Spain \\ ${ }^{2}$ Centro Superior de Estudios Felipe II, Ingeniería Técnica en informática de Sistemas \\ 28300 Aranjuez, Madrid, Spain \\ ${ }^{3}$ Dpto. Arquitectura Computadores y Automática, Facultad de Informática, \\ Universidad Complutense, 28040 Madrid, Spain \\ pjherrera@pdi.ucm.es, pajares@fdi.ucm.es, \\ mguijarro@cesfelipesegundo.com, \\ \{jjruz, jmcruz\} @dacya.ucm.es
}

\begin{abstract}
This paper describes a novel stereovision matching approach by combining several attributes at the pixel level for omni-directional images obtained with fish-eye lenses in forest environments. The goal is to obtain a disparity map as a previous step for determining distances to the trees and then the volume of wood in the imaged area. The interest is focused on the trunks of the trees. Because of the irregular distribution of the trunks, the most suitable features are the pixels. A set of six attributes is used for establishing the matching between the pixels in both images of the stereo pair. The final decision about the matched pixels is taken by combining the attributes. Two combined strategies are proposed: the Sugeno Fuzzy Integral and the Dempster-Shafer theory. The combined strategies, applied to our specific stereo vision matching problem, make the main finding of the paper. In both, the combination is based on the application of three well known matching constraints. The proposed approaches are compared among them and favourably against the usage of simple features.
\end{abstract}

Keywords: Sugeno Fuzzy Integral, Dempster-Shafer theory, fish-eye stereo vision, Stereovision matching, omni-directional forest images.

\section{Introduction}

One important task in forests maintenance is to determine the volume of wood in an area for different purposes, including the control of growth of the trees. This task can be carried out by stereovision systems. Fish-eye lenses allow imaging a large sector of the surrounding space with omni-directional vision. This justifies its use.

According to [1] we can view the classical problem of stereo analysis as consisting of the following steps: image acquisition, camera modelling, feature acquisition, image matching, depth determination and interpolation. The key step is that of image 
matching. This is the process of identifying the corresponding points in two images that are cast by the same physical point in the 3-D space. This paper is devoted solely to the matching one. Two sorts of techniques have been used for matching: area-based and feature based $[2,3]$.

Area-based stereo techniques [4] use correlation between brightness (intensities) patterns in the local neighbourhood of a pixel in one image with brightness patterns in the local neighbourhood of the other image. Also statistical textures can be considered under this category. Feature-based methods [5] use set of pixels with similar attributes, colour, gradient (module and direction) or Laplacian. These are the six attributes available to be used in our matching procedure.

Figure 1(a) displays one omni-directional image (let's say the left one) of the stereo pair captured with a camera equipped with a fish-eye lens. Figure 1 $(b)$ displays the signed and expanded area on Figure 1(a). In Figure 1 $(c)$ the corresponding area in the right image of the stereo pair is displayed. The different locations of the tree's crowns with respect each camera of the stereovision system produce an important lighting variability between both areas. This is applicable to the whole image. Moreover, as mentioned before, our interest is only focused on the matching of the trunks because they contain basically the wood. One could think about the matching of features such as the trunks themselves, perhaps by exploiting their forms and apparent orientation towards the centre of the image. But this is a complex task because depending on the sun position there are trunks fully and partially illuminated or in shade, this can be observed in the right or left semicircles in the image of figure $1 a$, respectively. Additionally, only exact vertical trees with respect the image system are imaged oriented toward the centre, but this rarely occurs, i.e. radial explorations do not follow exactly the trunks. Because of this difficulty the segmentation of the trunks as features has been postponed for future research. This is the reason by which this paper is focused on the matching at the pixel level.

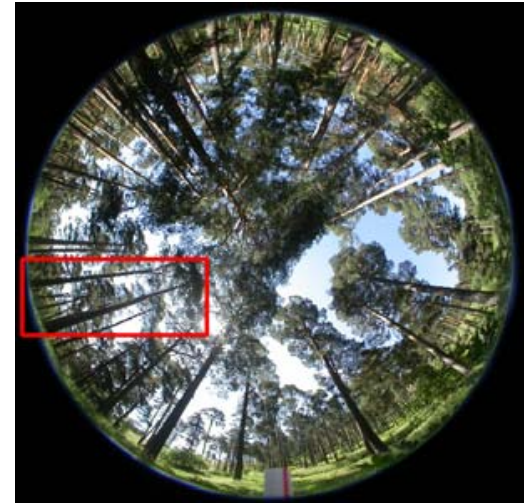

(a)

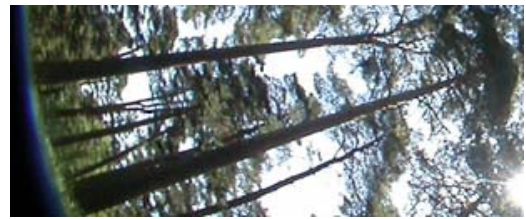

(b)

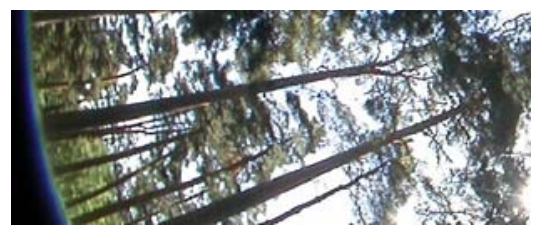

(c)

Fig. 1. (a) Omni-directional left image; (b) left expanded area; (c) corresponding right expanded area 
The following three stereovision constraints can be applied for solving the matching problem. Epipolar: derived from the system geometry, given a pixel in one image its correspondence will be on the called epipolar line. Similarity: matched pixels display similar attributes or properties. Uniqueness: a pixel in the left image must be matched to a unique pixel in the right one.

Given a pixel in the left image, we apply the epipolar constraint for determining a list of candidates, which are potential matches, in the right image. Each candidate becomes an alternative for the first pixel. We also apply the similarity constraint based on the six attributes mentioned above, obtaining six similarity measures. The final decision about the correct match, among the list of candidates, is made according to the support that each candidate receives by combining the similarity measures. Two strategies are used for the combination: the Sugeno Fuzzy Integral (SFI) paradigm and the Dempster-Shafer (DES) theory. The unique selection made in SFI or DES implies the application of the uniqueness constraint. The combined SFI and DES strategies make the main contribution of this paper. The proposed approaches are compared among them and favourably against the usage of individual attributes catalogued as area-based and feature-based correspondence techniques.

This work is organized as follows. Section 2 describes the design of the matching process; including a brief overview of the SFI paradigm and DES theory. Section 3 describes the results obtained by using the combined SFI and DES approaches, and comparing these results with those obtained by considering the attributes individually. Section 4 presents the conclusions and future work.

\section{Design of the Matching Process}

\subsection{Epipolar: System Geometry}

Figure 2 displays the stereo vision system geometry [6]. The 3D object point $P$ with world coordinates with respect to the systems $\left(X_{1}, Y_{1}, Z_{1}\right)$ and $\left(X_{2}, Y_{2}, Z_{2}\right)$ is imaged as $\left(x_{i 1}, y_{i 1}\right)$ and $\left(x_{i 2}, y_{i 2}\right)$ in image- 1 and image- 2 respectively in coordinates of the image system; $\alpha_{1}$ and $\alpha_{2}$ are the angles of incidence of the rays from $P ; y_{12}$ is the baseline measuring the distance between the optical axes in both cameras along the $y$-axes; $r$ is the distance between image point and optical axis; $R$ is the image radius, identical in both images.

According to [7], the following geometrical relations can be established,

$$
r=\sqrt{x_{i 1}^{2}+y_{i 1}^{2}} ; \alpha_{1}=\left(r 90^{\circ}\right) / R ; \beta=\operatorname{tg}^{-1}\left(y_{i 1} / x_{i 1}\right)
$$

Now the problem is that the $3 \mathrm{D}$ world coordinates $\left(X_{1}, Y_{1}, Z_{1}\right)$ are unknown. They can be estimated by varying the distance $d$ as follows,

$$
X_{1}=d \cos \beta ; \quad Y_{1}=d \sin \beta ; \quad Z_{1}=\sqrt{X_{1}^{2}+Y_{1}^{2}} / \tan \alpha_{1}
$$




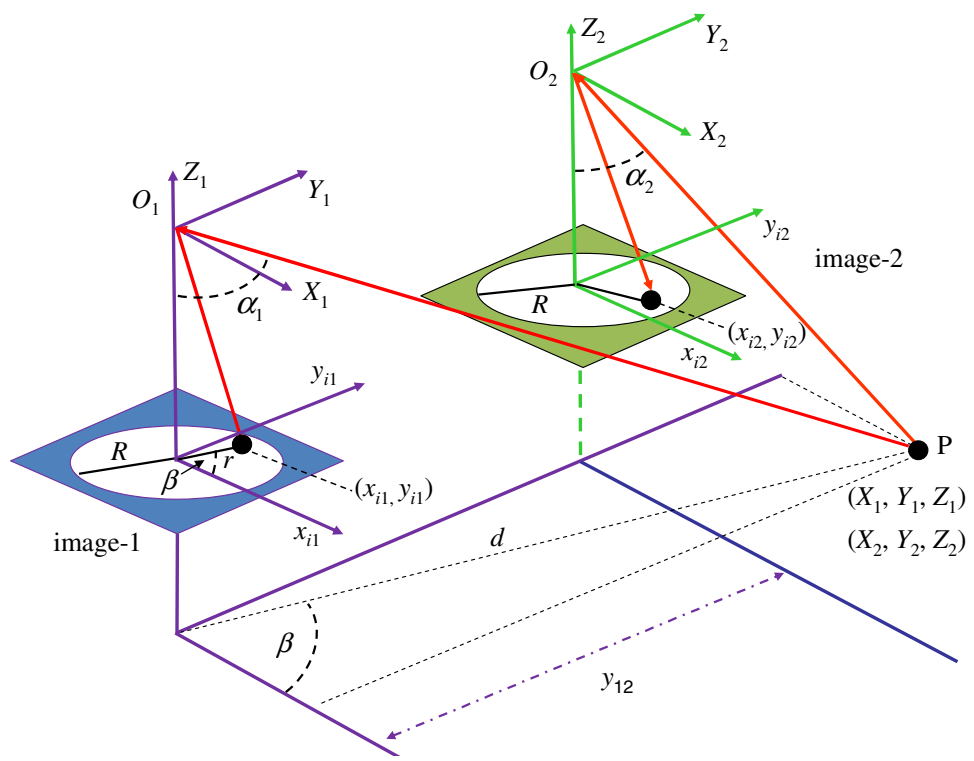

Fig. 2. Geometric projections and relations for the fish-eye based stereo vision system

From (2) we transform the world coordinates in the system $O_{1} X_{1} Y_{1} Z_{1}$ to the world coordinates in the system $\mathrm{O}_{2} \mathrm{X}_{2} Y_{2} \mathrm{Z}_{2}$ taking into account the baseline as follows:

$$
X_{2}=X_{1} ; \quad Y_{2}=Y_{1}+y_{12} ; \quad Z_{2}=Z_{1}
$$

Assuming no lenses radial distortion, we can find the imaged coordinates of the 3D point in image-2 as [7],

$$
x_{i 2}=\frac{2 R \arctan \left(\sqrt{X^{2}+Y^{2}} / Z_{2}\right)}{\pi \sqrt{\left(Y_{2} / X_{2}\right)^{2}+1}} ; \quad y_{i 2}=\frac{2 R \arctan \left(\sqrt{X^{2}+Y^{2}} / Z_{2}\right)}{\pi \sqrt{\left(X_{2} / Y_{2}\right)^{2}+1}}
$$

Because of the system geometry, the epipolar lines are not concentric circumferences and this fact is considered for matching. Figure 3 displays four epipolar lines, in the third quadrant of the right image, which have been generated by the four pixels located at the positions marked with the squares, which are their equivalent locations in the left image.

Using only a camera, we capture a unique image and each $3 \mathrm{D}$ point belonging to the line $\overline{O_{1} P}$, is imaged in $\left(x_{i 1}, y_{i 1}\right)$. So, the $3 \mathrm{D}$ coordinates with a unique camera cannot be obtained. When we try to match the imaged point $\left(x_{i 1}, y_{i 1}\right)$ into the image-2 we follow the epipolar line, i.e. the projection of $\overline{O_{1} P}$ over the image-2. This is equivalent to vary the parameter $d$ in the 3-D space. So, given the imaged point $\left(x_{i 1}, y_{i 1}\right)$ in the image-1 (left) and following the epipolar line, we obtain a list of $m$ 
potential corresponding candidates represented by $\left(x_{i 2}, y_{i 2}\right)$ in the image-2 (right). The best match is associated to a distance $d$ for the 3D point in the scene, which is computed from the stereo vision system. Hence, for each $d$ we obtain a specific $\left(x_{i 2}, y_{i 2}\right)$, so that when it is matched with $\left(x_{i 1}, y_{i 1}\right) d$ is the distance for the point $P$. Different measures of distances during different time intervals (years) for specific points in the trunks, such as the ends or the width of the trunk measured at the same height, allow determining the evolution of the tree and consequently its state of growth and also the volume of wood. This requires that the stereovision system is placed at the same position in the 3D scene and also with the same camera orientation (left camera North and right camera South).

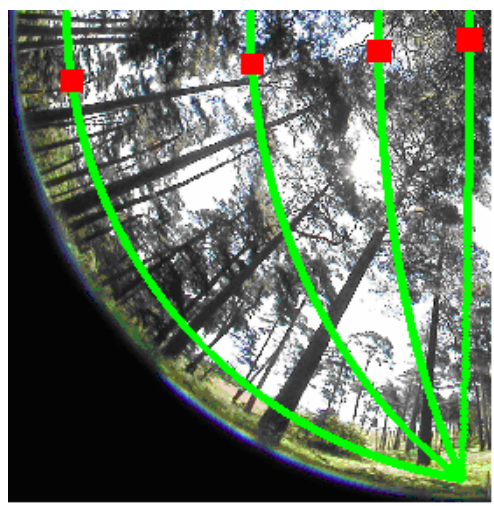

Fig. 3. Epipolar lines in the right image generated from the locations in the left image marked with the squares

\subsection{Similarity: Attributes for Area and Feature-Based}

Each pixel $l$ in the left image is characterized by its attributes; one of such attributes is denoted as $A_{l}$. In the same way, each candidate $i$ in the list of $m$ candidates is described by identical attributes, $A_{i}$. So, we can compute differences between attributes of the same type $A$, obtaining a similarity measure for each one as follows,

$$
s_{i A}=\left(1+\left|A_{l}-A_{i}\right|\right)^{-1} ; \quad i=1, \ldots, m
$$

$s_{i A} \in[0,1], s_{i A}=0$ if the difference between attributes is large enough (minimum similarity), otherwise if they are equal ( $s_{i A}=1$, maximum similarity).

As mentioned before, in this paper we use the following six attributes for describing each pixel: $a$ ) correlation; $b$ ) texture; $c$ ) colour; $d$ ) gradient magnitude; $e$ ) gradient direction and $f$ ) Laplacian. Both first ones are area-based computed on a $3 \times 3$ neighbourhood around each pixel through the correlation coefficient $[8,9,10]$ and standard deviation [11] respectively. The four remaining ones are considered as feature-based [5]. The colour involves the three red-green-blue spectral components $(\mathrm{R}, \mathrm{G}, \mathrm{B})$ and the absolute value in the equation (5) is extended as the sum of absolute 
differences as $\left|A_{l}-A_{i}\right|=\sum_{H}\left|H_{l}-H_{i}\right|, H=\mathrm{R}, \mathrm{G}, \mathrm{B}$. It is a similarity measurement for colour images [9], used satisfactorily in [10] for stereovision matching. Gradient (magnitude and direction) and Laplacian are computed by applying the first and second derivatives [11], over the intensity image after its transformation from the RGB plane to the HSI (hue, saturation, intensity) one. The gradient magnitude has been used in $[5,10]$ and the direction in [5]. Both, colour and gradient magnitude have been linearly combined in [10] producing satisfactory results as compared with the Middlebury test bed [3]. The coefficients involved in the linear combination are computed by testing reliable correspondences in a set of experiments carried out during a previous stage. Based on the conclusions reported in [10], the combination of attributes appears as a suitable approach. SFI and DES can cope specifically with the combination of attributes because they are specifically designed for classifier combination [12]. With a little adjusting they can be used for combining attributes in stereovision matching. They allow making a decision about a unique candidate (uniqueness constraint). This justifies their choice in this paper. We give details about them in section 2.3.

Given a pixel in the left image and the set of $m$ candidates in the right one, we compute the following similarity measures for each attribute $A$ : $s_{i a}$ (correlation), $s_{i b}$ (colour), $s_{i c}$ (texture), $s_{i d}$ (gradient magnitude), $s_{i e}$ (gradient direction) and $s_{i f}$ (Laplacian). The identifiers in the sub indices identify the attributes according to these assignments. The attributes are the six described above, i.e. $\Omega \equiv\{a, b, c, d, e, f\}$ associated to correlation, texture, colour, gradient magnitude, gradient direction and Laplacian.

\subsection{Uniqueness: Combined Strategies}

Now we must match each pixel $l$ in the left image with the best of the potential candidates (uniqueness). This is a decision that can be made through the combined SFI paradigm and the DES theory. We describe briefly both approaches.

\subsubsection{SFI: Sugeno Fuzzy Integral Paradigm}

This paradigm allows combining the individual similarities, which are computed through the equation (5). The SFI requires the computation of the relevance assigned for each attribute, from which we can compute the so-called fuzzy densities. This is solved by computing the $\lambda$-fuzzy measure using the data [12]. The calculation starts with selecting a set of six fuzzy values, $g^{\mathrm{a}}, \mathrm{g}^{\mathrm{b}}, \mathrm{g}^{\mathrm{c}}, \mathrm{g}^{\mathrm{d}}, \mathrm{g}^{\mathrm{e}}, \mathrm{g}^{\mathrm{f}}$, each one representing the individual relevance of the associated attribute in $\Omega$.

The value of $\lambda$ needed for calculating the fuzzy densities $g$ is obtained as the unique real root greater than -1 of the polynomial,

$$
\lambda+1=\prod_{j \in \Omega}\left(1+\lambda g^{j}\right)
$$

The individual relevancies for each attribute are computed from the data, as described later in the section 3.1 .

Once the $g^{a}, \ldots g^{f}$ are obtained and $\lambda$ is found, the SFI works as follows:

1. For a given pixel $l$ in the left image, we compute the similarities through the equation (5) between $l$ and every candidate $i$, with $i=1, \ldots, m$, obtaining a 
column vector as: $\left[s_{i a}, s_{i b}, s_{i c}, s_{i d}, s_{i e}, s_{i f}\right]^{\mathrm{T}}$; without lost of generality assume that $s_{i a}$ is the highest similarity value and $s_{i f}$ the lowest. This vector is arranged under this criterion, i.e. $s_{i a}>s_{i b}>s_{i c}>s_{i d}>s_{i e}>s_{i f}$.

2. Arrange the above fuzzy values correspondingly with the mentioned arrangement, i.e. $g^{a}, g^{b}, g^{c}, g^{d}, g^{e}, g^{f}$ and set the first fuzzy density $g(a)=g^{a}$.

3. Compute the remaining fuzzy densities,

$$
\begin{aligned}
g(b)= & g^{b}+g(a)+\lambda g^{b} g(a) \\
g(c)= & g^{c}+g(b)+\lambda g^{c} g(b) \\
& \ldots \ldots \ldots \ldots \ldots \ldots \ldots . . . \\
g(f)= & g^{f}+g(e)+\lambda g^{f} g(e)
\end{aligned}
$$

4. Calculate for each candidate $i$, the support to be matched with $l$ as,

$$
\mu_{i}(l)=\max _{h \in \Omega}\left\{\min \left\{s_{i h}, g(h)\right\}\right\}
$$

5. The decision about the best match is made by selecting the maximum support $\mu_{i}(l)$ among all candidates, but only if this value is greater than a threshold, set to 0.5 in this paper, after trial and error.

\subsubsection{DES: Dempster-Shafer Theory}

The Dempster-Shafer theory owes its name to works by the both authors in [13] and [14], the method as it is applied in our approach is as follows [12]:

1. In our stereovision matching problem a pixel $l$ is matched correctly or incorrectly. Hence, we identify two classes, which are the class of true matches and the class of false matches, $w_{1}$ (true) and $w_{2}$ (false). Given a set of samples from both classes, we compute the similarities of the matches belonging to each class according to (5) and build a 6-dimensional mean vector, where its components are the mean values of their similarities, i.e. $\overline{\boldsymbol{v}}_{j}=\left[\bar{s}_{j a}, \bar{s}_{j b}, \bar{s}_{j c}, \bar{s}_{j d}, \bar{s}_{j e}, \bar{s}_{j f}\right]^{\mathrm{T}} ; \overline{\boldsymbol{v}}_{1}$ and $\overline{\boldsymbol{v}}_{2}$ are the mean for $w_{1}$ and $w_{2}$ respectively. This is carried out during a previous phase, equivalent to the training one in classification problems.

2. Given a candidate $i$ from the list of $m$ candidates for $l$, we compute the 6 dimensional vector $\boldsymbol{x}_{i}$, where its components are the similarity values obtained according to (5) between $l$ and $i$, i.e. $\boldsymbol{x}_{i}=\left[s_{i a}, s_{i b}, s_{i c}, s_{i d}, s_{i e}, s_{i f}\right]^{\mathrm{T}}$. Then we calculate the proximity $\Phi$ between each component in $\boldsymbol{x}_{i}$ and each component in $\overline{\boldsymbol{v}}_{j}$ based on the Euclidean norm $\|\cdot\|$, equation (9).

$$
\Phi_{j A}\left(\boldsymbol{x}_{i}\right)=\frac{\left(1+\left\|s_{i A}-\bar{s}_{j A}\right\|^{2}\right)^{-1}}{\sum_{k=1}^{2}\left(1+\left\|s_{i A}-\bar{s}_{k A}\right\|^{2}\right)^{-1}} \text { where } A \in \Omega
$$


3. For every class $w_{j}$ and for every candidate $i$, we calculate the belief degrees,

$$
b_{j}^{i}(A)=\frac{\Phi_{j A}\left(x_{i}\right) \prod_{k \neq j}\left(1-\Phi_{k A}\left(x_{i}\right)\right)}{1-\Phi_{j A}\left(x_{i}\right)\left[1-\prod_{k \neq j}\left(1-\Phi_{k A}\left(x_{i}\right)\right)\right]} ; j=1,2
$$

4. The final degree of support that candidate $i$, represented by $\boldsymbol{x}_{i}$, receives for each class $w_{j}$ taking into account that its math is $l$ is given by,

$$
\mu_{j}\left(\boldsymbol{x}_{i}\right)=\prod_{A \in \Omega} b_{j}^{i}(A)
$$

5. We chose as the best match for $l$, the candidate $i$ with the maximum support received for the class of true matches $\left(w_{1}\right)$, i.e. $\max _{i}\left\{\mu_{1}\left(\boldsymbol{x}_{i}\right)\right\}$ but only if it is greater than a threshold set to 0.5 in this paper after experimentation, as in the SFI approach.

\section{Results}

The system is based on the scheme of the figure 2 , with a baseline of 1 meter. The cameras are equipped each one with Nikon FC-E8 fisheye lens, with an angle of $183^{\circ}$. The valid colour images in the circle contain 6586205 pixels.

The tests have been carried out with twenty pairs of stereo images. We use four of them for computing the relevance of each attribute for SFI, from which the fuzzy densities can be obtained, and the mean vectors $\overline{\boldsymbol{v}}_{1}$ and $\overline{\boldsymbol{v}}_{2}$ for DES. At a second stage, we apply the SFI and DES approaches pixel by pixel for the remainder sixteen stereo pairs, as described respectively in subsections 2.3.1 and 2.3.2.

Our interest consists of determining the disparity of the trees trunks located in an area of $25 \mathrm{~m}^{2}$ around the stereo vision system.

The disparity is the absolute difference value in sexagesimal degrees, taking into account the imaged circle, between the pixel in the left image and its matched pixel in the right one.

We have available the information of disparities provided by the end users. Thus, for each pixel in a trunk we know its correct disparity value according to this expert knowledge; which allows us to compute the percentage of error. For each one of the sixteen pairs of stereo images used for testing, we compute the disparity error for the trunks and then average these errors among the sixteen pairs of stereo images.

\subsection{Computing the Relevancies in SFI and the Mean Vectors for DES}

As mentioned before, SFI and DES are suitable for combining classifiers. Both require a previous training to learn some parameters. In classification, SFI learns the relevance for each classifier, so that, during the combination, every classifier intervenes with a specific different weight on the final decision. In our combined SFI stereovision matching approach, we also compute the relevance of each attribute for determining its specific contribution to the decision through the fuzzy densities. The original DES combiner uses decision templates for comparing the new patterns to be classified, in our stereovision matching approach the decision templates are replaced 
by the mean vectors $\overline{\boldsymbol{v}}_{1}$ and $\overline{\boldsymbol{v}}_{2}$. As in [10], although with a different criterion, these vectors and the relevancies are determined by considering a number of reliable true and false matches obtained from the set of four pairs of stereo images used for this purpose. This is carried out as follows, for each pixel in the left images, we compute the disparity with respect its matched pixel in the right ones, but considering each one of the six attributes separately through the equation (5). So, we compute the averaged percentage of error for the four pairs of stereo images and for each attribute, based on the expert knowledge available about the disparities in the trunks. These percentages are: $p_{a}=28$ (correlation), $p_{b}=10$ (colour), $p_{c}=14$ (texture), $p_{d}=9$ (gradient magnitude), $p_{e}=30$ (gradient direction) and $p_{f}=27$ (Laplacian). So, the individual relevancies are computed as $g^{h}=p_{h} / \sum_{k} p_{k}, h, k=a, b, c, d, e, f$. Finally, the fuzzy values are exactly the following: $\mathrm{g}^{a}=0.150, g^{b}=0.179, g^{c}=0.187, g^{d}=0.189$, $g^{e}=0.145$ and $g^{f}=0.152$. As one can see, the most relevant attribute is the gradient magnitude.

Also, considering the true and false matches under the expert knowledge, we compute for each attribute the similarity values according to equation (5) and average them for true and false matches, obtaining: $\overline{\boldsymbol{v}}_{1}=[0.81,0.85,0.92,0.96,0.79,0.80]^{\mathrm{T}}$ and $\overline{\boldsymbol{v}}_{2}=[0.12,0.11,0.08,0.07,0.11,0.10]^{\mathrm{T}}$ respectively.

\subsection{SFI and DES Performances}

As before, for each pixel in each one of the sixteen pairs of stereo images, available for testing, we obtain its disparity considering the six attributes separately by applying the equation (5) and a maximum similarity criterion among the $m$ candidates and also by applying the SFI and DES approaches based on maximum supports, given by equations (8) and (11) respectively.

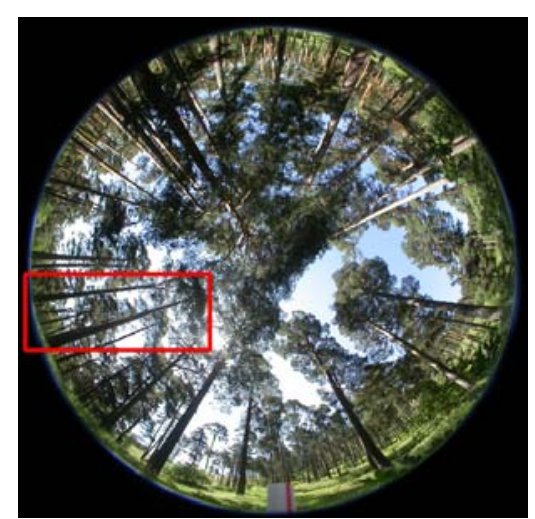

(a)

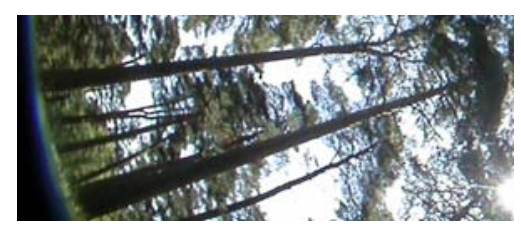

(b)

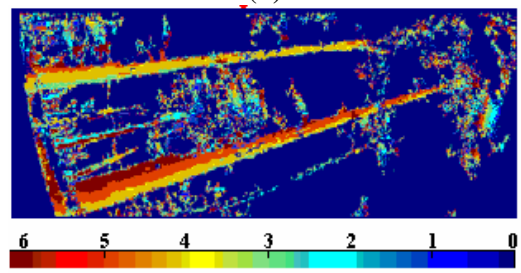

(c)

Fig. 4. (a) Left image; (b) expanded area; (c) disparity map obtained by the SFI approach 
Figures $4(a)$ and $4(b)$ are the same that Figures $1(a)$ and $1(b)$ respectively. Figure $4(c)$ displays the disparity map obtained by the SFI approach in the area. The colour bar shows the disparity level values according to the colour. The disparity map obtained by DES is very similar.

Table 1 displays the averaged percentage of error based on the similarity for the six individual attributes used separately, identified as: $\left(s_{a}, s_{b}, s_{c}, s_{d}, s_{e}, s_{f}\right)$. The averaged percentage of error obtained with both, SFI and DES decision making approaches are also displayed. The standard deviations are included.

Table 1. Averaged percentage of errors and standard deviations obtained through maximum similarity criteria for each attribute separately and the SFI and DES decision making approaches

\begin{tabular}{|c|c|c|c|c|c|c|c|c|c|c|c|c|c|c|c|}
\hline \multicolumn{11}{|c|}{ Averaged percentage of error and standard deviations } \\
\hline \multicolumn{2}{|c|}{$s_{a}$} & \multicolumn{2}{|c|}{$s_{b}$} & \multicolumn{2}{|c|}{$s_{c}$} & \multicolumn{2}{|c|}{$s_{d}$} & \multicolumn{2}{|c|}{$s_{e}$} & \multicolumn{2}{|c|}{$s_{f}$} & \multicolumn{2}{|c|}{ DES } & \multicolumn{2}{c|}{ SFI } \\
\hline$\%$ & $\sigma$ & $\%$ & $\sigma$ & $\%$ & $\sigma$ & $\%$ & $\sigma$ & $\%$ & $\sigma$ & $\%$ & $\sigma$ & $\%$ & $\sigma$ & $\%$ & $\sigma$ \\
\hline 30 & 2.9 & 16 & 1.3 & 18 & 1.7 & 14 & 1.1 & 35 & 3.6 & 32 & 3.1 & $\mathbf{1 1}$ & $\mathbf{1 . 6}$ & $\mathbf{1 1}$ & $\mathbf{1 . 3}$ \\
\hline
\end{tabular}

From results in table 1, one can see that both SFI and DES obtain similar results and outperform the individual similarity based approaches. This means that the combination of similarities between attributes improve the results obtained by using similarities separately. The best individual similarity results are obtained through the similarities provided by the gradient magnitude attribute $\left(s_{d}\right)$. This implies that it is the most relevant attribute. This agrees with its relevance obtained in section 3.1, as it has turned out to be the most relevant attribute.

\section{Concluding Remarks}

In this paper we have proposed a strategy for stereovision matching, with omnidirectional images, in a system equipped with fish-eye lenses. The method applies three well-known constraints (epipolar, similarity and uniqueness) by combining area-based and feature-based matching attributes. For each pixel in the left image a list of possible candidates in the right image is obtained for determining its correspondence. The similarity between attributes establishes measures for the matching between each left pixel and its candidates. Under the SFI paradigm and the DES theory, we combine the similarities between six attributes and make a decision for choosing the unique candidate, if any, for each left pixel. The proposed combined strategies obtain similar results and outperform the methods that use similarities separately.

Although the results achieved can be considered satisfactory, they could be improved by applying additional constraints such as smoothness or ordering, which have been used for matching in conventional stereovision systems.

The proposed combined approaches can be applied to any environment for stereovision matching, i.e. obviously classical ones with images captured with lenses under perspective projection. The unique modification consists in the computation of the 
epipolar lines, which determine where the candidate pixels must be located. In classical stereovision system they are straight and horizontal lines.

\section{Acknowledgements}

The authors wish to acknowledge to the Council of Education of the Autonomous Community of Madrid and the Social European Fund for the research contract with the first author. Also to Dr. Fernando Montes, from the Escuela Técnica Superior de Ingenieros de Montes at the Politechnical University of Madrid and to Dra. Isabel Cañellas from the Forest Research Centre (CIFOR, INIA) for his support and the imaged material supplied. To the DPI2006-15661-C02-01 project, a part of the research has been granted by it. Finally, to the anonymous three referees for their valuable comments and suggestions.

\section{References}

1. Barnard, S., Fishler, M.: Computational Stereo. ACM Computing Surveys 14, 553-572 (1982)

2. Cochran, S.D., Medioni, G.: 3-D Surface Description from binocular stereo. IEEE Trans. Pattern Anal. Machine Intell. 14(10), 981-994 (1992)

3. Scharstein, D., Szeliski, R.: A taxonomy and avaluation of dense two-frame stereo correspondence algorithms. Int. J. Computer Vision 47(1-3), 7-42 (2002),

http://vision.middlebury.edu/stereo/

4. Tang, L., Wu, C., Chen, Z.: Image dense matching based on region growth with adaptive window. Pattern Recognit. Lett. 23, 1169-1178 (2002)

5. Lew, M.S., Huang, T.S., Wong, K.: Learning and feature selection in stereo matching. IEEE Trans. Pattern Anal. Machine Intell. 16, 869-881 (1994)

6. Abraham, S., Förstner, W.: Fish-eye-stero calibration and epipolar rectification. Photogrammetry and Remote Sensing 59, 278-288 (2005)

7. Schwalbe, E.: Geometric Modelling and Calibration of Fisheye Lens Camera Systems. In: Proc. 2nd Panoramic Photogrammetry Workshop, Int. Archives of Photogrammetry and Remote Sensing, vol. 36, Part 5/W8 (2005)

8. Barnea, D.I., Silverman, H.F.: A Class of Algorithms for Fast Digital Image Registration. IEEE Trans. Computers 21, 179-186 (1972)

9. Koschan, A., Abidi, M.: Digital Color Image Processing. Wiley, Chichester (2008)

10. Klaus, A., Sormann, M., Karner, K.: Segmented-Based Stereo Matching Using Belief Propagation and Self-Adapting Dissimilarity Measure. In: Proc. of 18th Int. Conference on Pattern Recognition, vol. 3, pp. 15-18 (2006)

11. Pajares, G., de la Cruz, J.M.: Visión por Computador: Imágenes digitales y aplicaciones, RA-MA (2007)

12. Kuncheva, L.: Combining Pattern Classifiers: Methods and Algorithms. Wiley, Chichester (2004)

13. Dempster, A.P.: A generalization of Bayesian inference. Journal of the Royal Statistical Society B 30, 205-247 (1968)

14. Shafer, G.: A Mathematical Theory of Evidence. Princeton University Press, Princeton (1976) 